\title{
COGROWTH OF REGULAR GRAPHS
}

\author{
S. NORTHSHIELD
}

\author{
(Communicated by Lawrence F. Gray)
}

\begin{abstract}
Let $\mathscr{G}$ be a $d$-regular graph and $T$ the covering tree of $\mathscr{G}$. We define a cogrowth constant of $\mathscr{G}$ in $T$ and express it in terms of the first eigenvalue of the Laplacian on $\mathscr{G}$. As a corollary, we show that the cogrowth constant is as large as possible if and only if the first eigenvalue of the Laplacian on $\mathscr{G}$ is zero. Grigorchuk's criterion for amenability of finitely generated groups follows.
\end{abstract}

In this note, we shall relate the first eigenvalue of the Laplacian on a connected regular graph to the size of the kernel of the universal covering map. The main results have been proven in [C, G, P]. The proof presented here appears simpler; it depends on the explicit formula for minimal positive solutions of $\Delta F+\varepsilon F=-I$.

Let $\mathscr{G}$ be a connected simple graph with constant vertex degree $d \geq 3$, $T$ be the universal covering tree of $\mathscr{G}$, and $\theta$ the covering map (i.e., $\theta$ is a vertex surjection of $T$ on $\mathscr{G}$ that preserves adjacency and vertex degree). We let $T$ and $\mathscr{G}$ denote the vertex sets of the corresponding graphs. Note that $T$ has constant vertex degree $d$. Since $T$ is connected, $T$ may be considered a metric space with the usual graph metric $\delta(\delta(x, y)$ is the length of the shortest path connecting $x$ and $y$ ). For $x \in T$ and $n \geq 0$, let $[x]=\theta^{-1}(\theta(x))$ and $S_{n}(x)=\{y: \delta(x, y)=n\}$. For $x, y \in T$, note that

$$
\limsup _{n \rightarrow \infty}\left|[y] \cap S_{n}(x)\right|^{1 / n}=\inf \left\{\lambda>0: \sum_{z \in[y]} \lambda^{-\delta(x, z)}<\infty\right\}
$$

and is thus independent of $x$ and $y$. We call this number, $\operatorname{cogr}(T, \mathscr{G})$, the cogrowth constant of $\mathscr{G}$ in $T$.

For $x, y$ vertices of a graph, we write $x E y$ if $x$ and $y$ are connected by an edge. For $x, y \in T$, let

$$
q(x, y)= \begin{cases}\frac{1}{d} & \text { if } x E y, \\ 0 & \text { otherwise. }\end{cases}
$$

Note that $q$ is the transition matrix of the simple random walk on $T$. Let $q^{(n)}$ denote the $n$th power of $q$. For $a, b \in \mathscr{G}$ and $x \in \theta^{-1}(a)$, since $\theta$ takes the

Received by the editors January 25, 1991.

1980 Mathematics Subject Classification (1985 Revision). Primary 60J15; Secondary 05C05, $05 \mathrm{C} 25,43 \mathrm{~A} 07$.

Key words and phrases. Regular graph, covering tree, amenable group, random walk. 
simple random walk on $T$ to the simple random walk on $\mathscr{G}$,

$$
p^{(n)}(a, b)=\sum_{y \in \theta^{-1}(b)} q^{(n)}(x, y)
$$

where $p$ is the transition matrix of the simple random walk on $\mathscr{G}$. We define $\Delta \equiv p-1, G \equiv \sum_{n \geq 0} p^{(n)}$, and for $\varepsilon \geq 0, G^{\varepsilon} \equiv \sum_{n \geq 0} p^{(n)} /(1-\varepsilon)^{n+1}$. Similarly, we define $\Delta_{T}, F$, and $F^{\varepsilon}$ as above with $p$ replaced by $q$.

$\Delta$ and $\Delta_{T}$ are the Laplacians on $\mathscr{G}$ and $T$, respectively. We call $\lambda_{\mathscr{G}}$ and $\lambda_{T}$ the first eigenvalues of $\Delta$ and $\Delta_{T}$, respectively. Since $T$ is $d$-regular,

$$
\lambda_{T}=1-2(d-1)^{1 / 2} / d
$$

(see $[\mathrm{DK}])$. Also,

$$
\lambda_{\mathscr{G}}=\sup \{\lambda: \exists f>0: \Delta f+\lambda f \leq 0\}
$$

(see [DK] or [N]). It is true that $\lambda_{\mathscr{G}} \leq \lambda_{T}$ (see [N]).

For $\varepsilon \leq \lambda_{T}$, let $a(\varepsilon)=d(1-\varepsilon) /(d-1), b=1 /(d-1)$, and $\sigma(\varepsilon) \leq \tau(\varepsilon)$ be the (real) roots of $t=a(\varepsilon)-b / t$. Note that

$$
\begin{aligned}
\sigma(\varepsilon) & =\left\{d(1-\varepsilon)-\left[d^{2}(1-\varepsilon)^{2}-4 d+4\right]^{1 / 2}\right\} / 2(d-1), \\
\tau(\varepsilon) & =\left\{d(1-\varepsilon)+\left[d^{2}(1-\varepsilon)^{2}-4 d+4\right]^{1 / 2}\right\} / 2(d-1) .
\end{aligned}
$$

In particular, $\sigma(\varepsilon)$ is increasing and $\tau(\varepsilon)$ is decreasing on $\left[0, \lambda_{T}\right)$.

Lemma. For $\varepsilon \in\left[0, \lambda_{T}\right), F^{\varepsilon}(x, y)=\sigma(\varepsilon)^{\delta(x, y)} /(1-\varepsilon-\sigma(\varepsilon))$.

Proof. Let $\varepsilon \in\left[0, \lambda_{T}\right)$. For $\lambda \in\left(\varepsilon, \lambda_{T}\right)$, there exists a function $f>0$ such that $\Delta_{T} f+\lambda f \leq 0$. Let $v=-\left(\Delta_{T} f+\varepsilon f\right) /(1-\varepsilon)$ and $r=q /(1-\varepsilon)$. Note that $v>0$ and $f=v+q f$. By induction, $f=\sum_{0 \leq k \leq n} r^{(k)} v+r^{(n+1)} f \geq$ $\sum_{0 \leq k \leq n} r^{(k)} v$ since $f>0$. Letting $n \rightarrow \infty, f \geq \sum_{k \geq 0} r^{(k)} v=(1-\varepsilon) F^{\varepsilon} v$. Since $v>0, F^{\varepsilon}$ exists.

By the symmetry of $T$, there exists a sequence $\gamma_{0}, \gamma_{1}, \ldots$ such that for any $x$, if $\delta(x, y)=n$ then $F^{\varepsilon}(x, y)=\gamma_{n}$. Since $\left(\Delta_{T}+\varepsilon\right) F^{\varepsilon}=-I$, it follows that $\gamma_{k+2}=a \gamma_{k+1}-b \gamma_{k}$ and $\gamma_{1}-(1-\varepsilon) \gamma_{0}=-1$. Let $r_{k}=\gamma_{k+1} / \gamma_{k}, \mu=a / 2$, and $\nu=\left[a^{2} / 4-b\right]^{1 / 2}$. By the addition of angle formulae for hyperbolic functions, it is easy to verify that for all $r_{0}$ there exists $\theta$, so that

$$
r_{n}= \begin{cases}\mu+\nu \tanh (\theta+\rho n) & \text { if } r_{0} \in(\sigma, \tau), \\ \mu+\nu \operatorname{coth}(\theta+\rho n) & \text { if } r_{0} \notin[0, \tau], \\ r_{0} & \text { if } r_{0} \in\{\sigma, \tau\},\end{cases}
$$

where $\rho=\tanh (\nu / \mu)$.

Clearly, if $r_{0} \neq \sigma$ then $r_{n} \rightarrow \tau$, and thus $\lim _{n \rightarrow \infty} \gamma_{n}^{1 / n}=\tau$. It is easy to verify that $\lim _{n \rightarrow \infty} \gamma_{n}^{1 / n}$ is increasing as a function of $\varepsilon$ (since $p^{(n)} \geq 0$ ). Therefore $r_{n} \equiv \sigma$ since $\tau$ is decreasing. It follows that $F^{\varepsilon}(x, y)=c \sigma^{\delta(x, y)}$ and, since $\tau_{1}-(1-\varepsilon) \gamma_{0}=-1, c=1 /[1-\varepsilon-\sigma]$.

Theorem. (a) $\operatorname{cogr}(T, \mathscr{G}) \leq\left\{d\left(1-\lambda_{\mathscr{G}}\right)+\left[d^{2}\left(1-\lambda_{\mathscr{G}}\right)^{2}-4 d+4\right]^{1 / 2}\right\} / 2$,

(b) If $\lambda_{\mathscr{G}} \neq \lambda_{T}$ then $\operatorname{cogr}(T, \mathscr{G})=\left\{d\left(1-\lambda_{\mathscr{G}}\right)+\left[d^{2}\left(1-\lambda_{\mathscr{G}}\right)^{2}-4 d+4\right]^{1 / 2}\right\} / 2$. Proof. (a) If $\lambda_{\mathscr{G}}=0$ then $\operatorname{cogr}(T, \mathscr{G}) \leq \lim \sup _{n \rightarrow \infty}\left|S_{n}(x)\right|^{1 / n}=d-1=$ $1 / \sigma\left(\lambda_{\mathscr{G}}\right)$. 
Let $\lambda_{\mathscr{G}}>0$ and $\varepsilon \in\left(0, \lambda_{\mathscr{G}}\right)$. As in the proof of the lemma, $G^{\varepsilon}$ exists. Since

$$
\sum_{z \in[y]} \sigma^{\delta(x, z)}=c \sum_{z \in[y]} F^{\varepsilon}(x, z)=c G^{\varepsilon}(\theta(x), \theta(y))<\infty,
$$

$\operatorname{cogr}(T, \mathscr{G}) \leq 1 / \sigma(\varepsilon)$. Since $\sigma(\varepsilon)$ is increasing, the result follows by letting $\varepsilon$ approach $\lambda_{\mathscr{G}}$.

(b) Let $\varepsilon \in\left[0, \lambda_{T}\right)$. If $\operatorname{cogr}(T, \mathscr{G})<1 / \sigma(\varepsilon)$, then

$$
G^{\varepsilon}(\theta(x), \theta(y))=\sum_{z \in[y]} F^{\varepsilon}(x, z)=\sum_{z \in[y]} \sigma^{\delta(x, z)}<\infty
$$

and thus $G^{\varepsilon}$ exists. Fix $g \in \mathscr{G}$ and let $f(x)=G^{\varepsilon}(g, x)$. Clearly $\Delta_{T} f+\varepsilon f \leq 0$ and $f>0$ and, therefore, $\varepsilon \leq \lambda_{\mathscr{G}}$. Assume $\lambda_{\mathscr{G}} \neq \lambda_{T}$ (and thus $\lambda_{\mathscr{G}}<\lambda_{T}$ ). If $\lambda_{\mathscr{G}}<\lambda_{\mathscr{G}}+\kappa \leq \lambda_{T}$, then $\operatorname{cogr}(T, \mathscr{G}) \geq 1 / \sigma\left(\lambda_{\mathscr{G}}+\kappa\right)$. Since $1 / \sigma$ is decreasing on $\left[0, \lambda_{\mathscr{G}}\right], \operatorname{cogr}(T, \mathscr{G}) \geq 1 / \sigma\left(\lambda_{\mathscr{G}}\right)$.

Corollary 1. Let $\mathscr{G}$ be connected and $d$-regular. Then $\operatorname{cogr}(T, \mathscr{G})=d-1$ iff $\lambda_{\mathscr{G}}=0$.

Let $A$ be a finitely generated discrete group with $k$ generators, $F$ the free group with $k$ generators, $\phi$ the canonical mapping of $F$ onto $A$, and $K=$ $\operatorname{ker} \theta$.

The map $\phi$ induces a covering map $\theta$ from $T$ onto $\mathscr{G}$ where $T$ and $\mathscr{G}$ are the Cayley graphs of $F$ and $A$ respectively. As is well known, $A$ is amenable iff $\lambda_{\mathscr{G}}=0$ (see [K, DK, DG]).

By [P], $\lim _{n \rightarrow \infty}\left|K \cap S_{2 n}\right|^{1 / 2 n}$ exists.

Corollary 2. $A$ is amenable iff $\lim _{n \rightarrow \infty}\left|K \cap S_{2 n}\right|^{1 / 2 n}=2 k-1$.

\section{REFERENCES}

[C] J. L. Cohen, Cogrowth and amenability of discrete groups, J. Funct. Anal. 48 (1982), 301309.

[DG] Y. Derriennic and Y. Guivarc' $h$, Theoreme de renouvellement pour les groupes moyennables, C. R. Acad. Sci. Paris Ser. A 277 (1973), 613-615.

[DK] J. Dodziuk and L. Karp, Spectral and function theory of combinatorial Laplacians, Contemp. Math., vol. 73, Amer. Math. Soc., Providence, RI, 1988, pp. 25-40.

[G] R. I. Grigorchuk, Symmetric random walks on discrete groups, Multi-Component Random Systems (Dobrushin and Sinai, eds.), Dekker, New York and Basel, 1980, pp. 285-325.

[K] H. Kesten, Full Banach mean values on countble groups, Math. Scand. 7 (1959), 146-156.

[N] S. Northshield, Gauge and conditional gauge on negatively curved graphs, Stochastic Anal. Appl. 9 (1991), 461-482.

[P] A. T. Paterson, Amenability, Math. Surveys Monographs, vol. 29, Amer. Math. Soc., Providence, RI, 1988.

Department of Mathematics, State University of New York at Plattsburgh, PlattsBURGH, NEW YORK 12901

E-mail address: northssw@snyplava.bitnet 